\title{
Control of droplet size in rain-zone in wet cooling tower
}

\author{
Rut Vitkovicova $^{1^{*}}$, Pavol Vitkovic ${ }^{1}$ \\ ${ }^{1}$ Czech Technical University in Prague, Department of Fluid Dynamics and Thermodynamics, 16607 Prague, Czech Republic
}

\begin{abstract}
The performance of the wet cooling tower is significantly affected by the droplet size occurring in the rain zone. In order to effectively manage the size of these droplets, it was necessary to experimentally determine the effect of the fills of the cooling towers on droplets. Five types of cooling fillers were used for experimental measurements: 3 film fills and 2 splash fills - trickle and grid. Drop size measurements were performed using the LIF method. Histograms of droplets size were obtained from measured droplet sizes under each fill, and for each fill, the Sauter droplet diameter was then calculated. According to a theoretical analysis of a breakdown of droplets, the combinations of some fills and the effect of their surface treatment on the droplet diameter were then measured for comparison.
\end{abstract}

\section{Introduction}

The task of the cooling tower is to dissipate away waste heat to the atmosphere [1]. In the wet cooling towers, cooled water is piped to nozzles that spray water on the cooling fills [2]. Significant heat dissipation occurs in three areas: in the area between the nozzles and the fill zone - the spray zone, the cooling fill itself, and the area under the cooling fills - the rain zone. The most significant heat transfer takes place directly on the cooling fills. The type of cooling fill determines how significant the heat transfer will be directly on the fill but also in the rain zone. Its mathematical model is described in [3] and [4].

There are many types of cooling fills, but the most common fills are film fills and splash fills (Figures 1-3). In the first type, as suggested by the name, water after impact on the surface of the fill creates a thin film of water, which allows to intensify the transfer of heat. The droplets under the fill are consequently mainly formed by dripping - drip effect. The second type of fill is based on breaking big water structures into smaller droplets splash effect. With the splash mechanism, a large number of small droplets can be obtained, thereby increasing the heat transfer surface. Film fills achieve greater efficiency, but the advantage of splash fills lies in their resistance to the influence of dirty water.

In order to effectively control the droplet size in the rain zone and hence the efficiency of cooling, it is necessary to know the range of droplet sizes formed under the individual fills. Consequently, experiments were carried out to determine droplet size in the rain zone for several types of fills. Based on these measurements and the theoretical analysis of the principle of droplet formation under cooling fills, two variants of fills were proposed to improve the heat transfer efficiency in the rain zone. The effect of these proposals was subsequently verified experimentally.

\section{Measurements}

\subsection{Measured fills}

The measurement of droplet size under the fill was performed for 5 commonly used fills. Three fills were the film fill type (Fig.1): a straight film fill, a slope film fill and an offset film fill. These fills are made up of blocks and the water runs down through the channel on the surface of the fill. The three measured film fills varied in the shape of this channel and its orientation in the fill. The straight film fill has a channel axis parallel to the direction of flow, the axis of the channel of the slope film fill is inclined to the direction of flow and the offset film fill has a curved channel.

The splash fills that were measured were a trickle and a grid type. The trickle fill consists of a dense network block (Fig 2).

The grid fill (Fig. 3) is also made up of a mesh, but not as dense as the trickle fill has it. The grid fill does not form a compact block, but it is composed of layers of individual panels. One layer was measured in this experiment.

\footnotetext{
* Corresponding author: rut.vitkovicova@fs.cvut.cz
} 


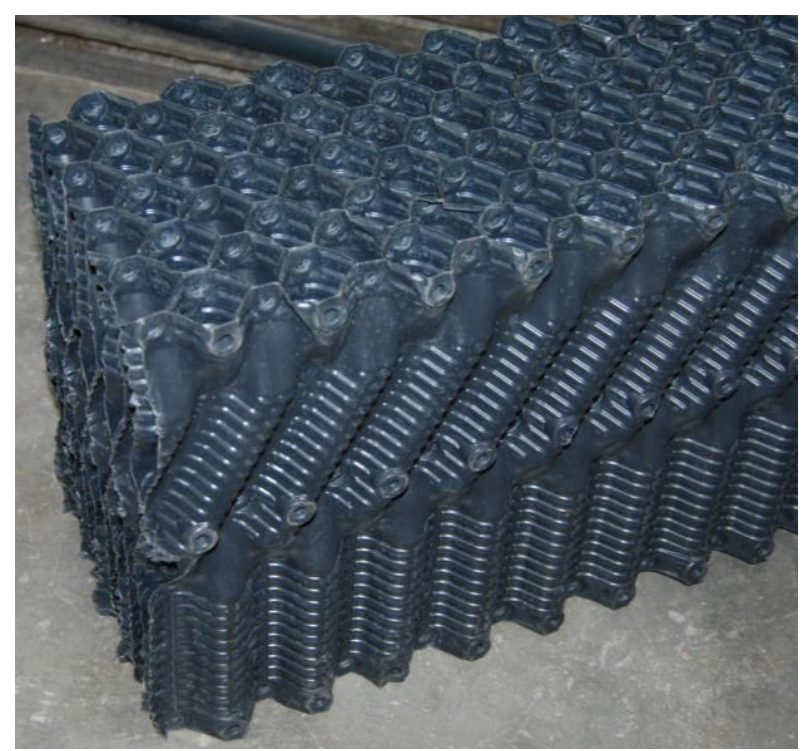

Fig. 1. Film fill

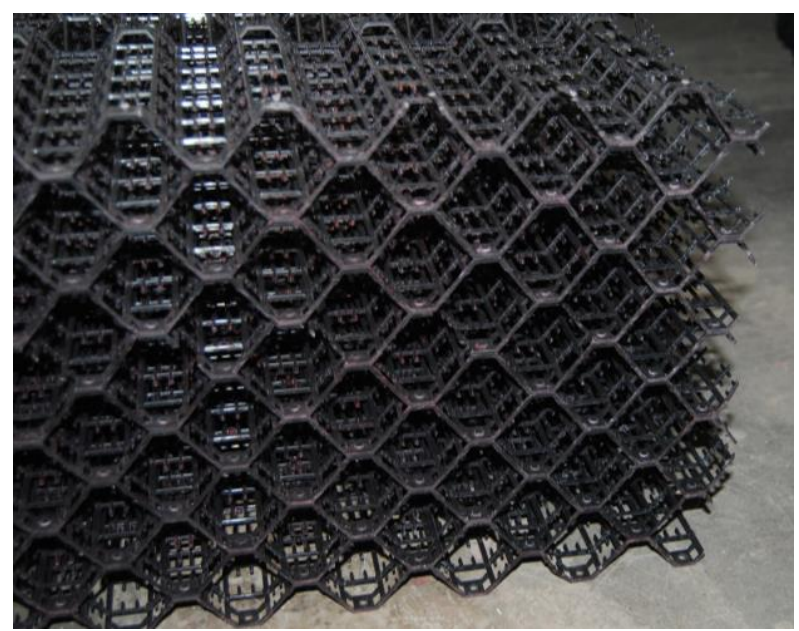

Fig. 2. Trickle fill

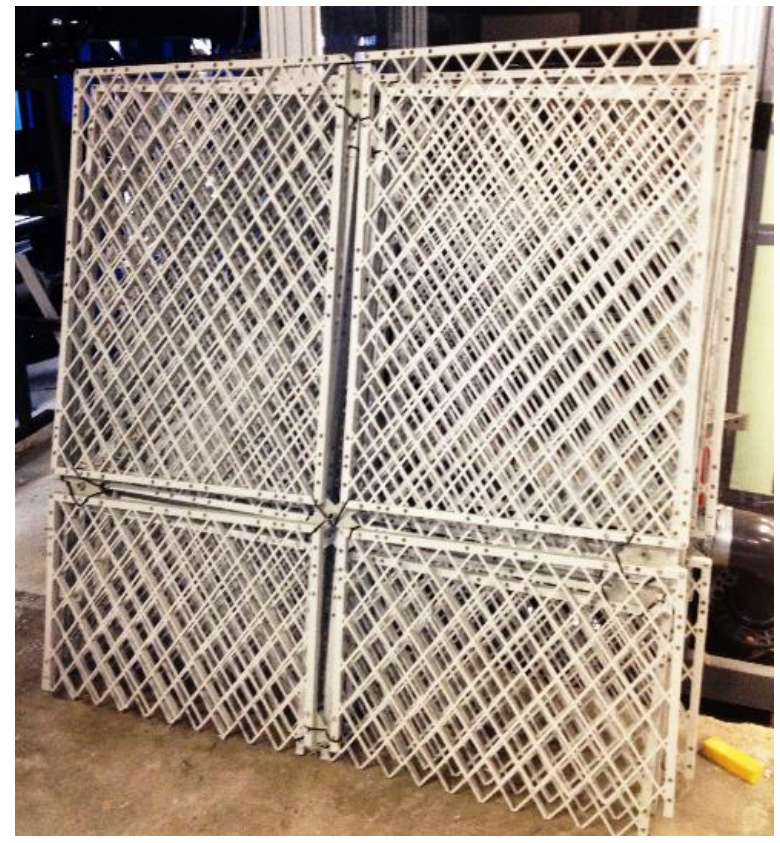

Fig. 3. Grid fill

\subsection{Experimental method and measurement facilities}

The Laser-Induced Fluorescence (LIF) method is used for obtaining a size of droplets below the fills. LIF has excellent detection sensitivity because a signal is observed against a dark background [5]. Main components of LIF system are a laser, camera with an optical filter and some fluorescent dye. Fluorescent solution (in this case Rhodamine B) in water can absorb light (green light) at one frequency and subsequently reemit light at a different frequency. The laser beam is expanded into a sheet using two cylindrical lenses. The cylindrical lens produces a light sheet of few millimeter thick. Rhodamine $\mathrm{B}$, as a tracer dye, emits light of wavelength between 500 and $700 \mathrm{~nm}$ after absorbing light with the wavelength of $532 \mathrm{~nm}$. Excitation light was filtered by a red filter with the cut-on wavelength about $600 \mathrm{~nm}$. All the reflections and other droplets that are in front and behind the light sheet are eliminated through the filter. The red filter was installed at the lens of a camera. In Fig. 4 is shown scheme of this used method.

Laser

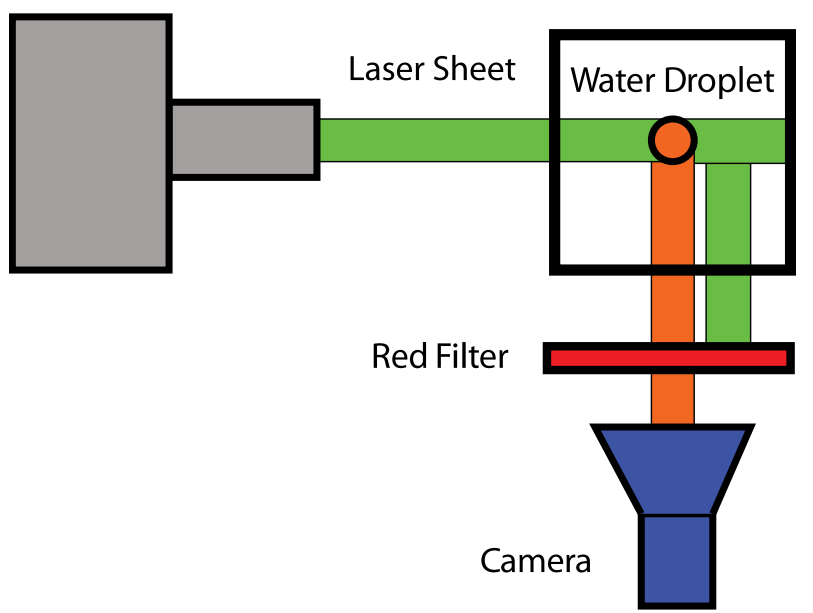

Fig. 4. LIF measurement

The experimental facility is consisting of a basin, a test section, an extending part, a water pump, a flow meter and a droplet generator. The test section is made from a plexiglass (Fig. 5). The dimension of the test section is $0.6 \times 0.6 \mathrm{~m}$ and a total height of this section is $1 \mathrm{~m}$. The extending part is consisting of several modules with a height of $1 \mathrm{~m}$ and $0.5 \mathrm{~m}$. 


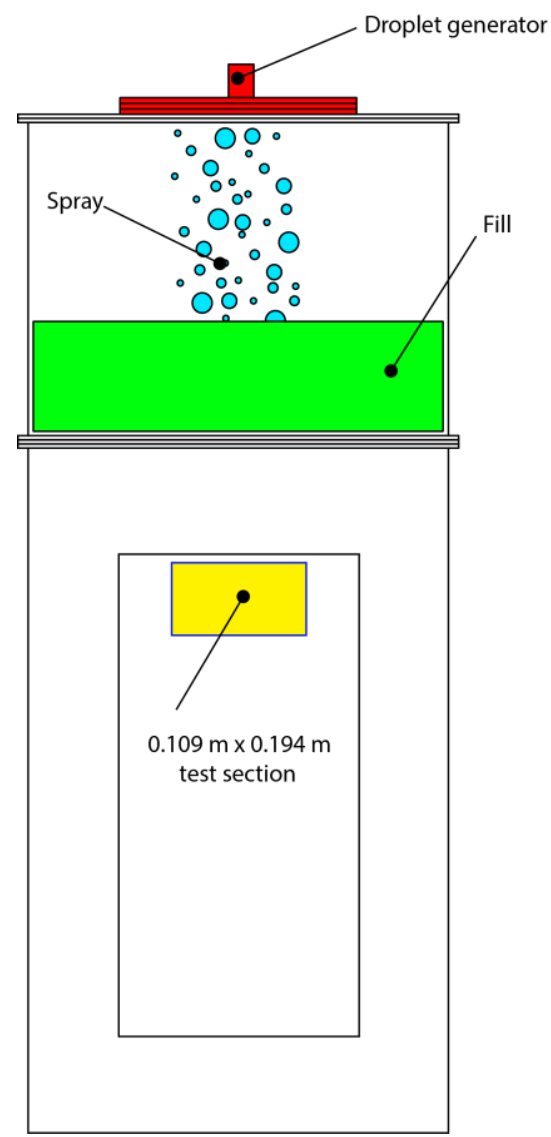

Fig. 5. Experimental facility

The modules are made from a galvanized metal sheet. And it can be arbitrarily combined with each other. The water droplets generator is supplied by the water feed pump connected to the water basin below the test section. The water flow rate is measured by the electromagnetic flow meter. The droplets generator was made from plexiglass sheets [6]. It is consisting of a three layers. The last layer has holes with $1 \mathrm{~mm}$ diameter arranged into the grid $0.01 \times 0.01 \mathrm{~m}$. The grid of the holes is creating an active zone in the test section. It dimension is $0.2 \times 0.2 \mathrm{~m}$. The droplets generator was placed on the top of the experimental facility. Droplets size distribution of droplet generator is based on [6].

\subsection{Set - up of experiments}

In this case, it was used measuring system which consist of the industry camera SONY XCG/H280E with $85 \mathrm{~mm}$ lens. The exposure time was set to $1 / 50 \mathrm{sec}$. The aperture was set at 1.8. The Coherent Verdi G Series semiconductor laser was used. The laser power was set at $1 \mathrm{~W}$.

The water flow rate was $11 \mathrm{l} / \mathrm{s}$. It was measured 4 distances $(1 \mathrm{~m}, 2 \mathrm{~m}, 3, \mathrm{~m}, 4 \mathrm{~m})$ between the bottom of the fill and test section for 5 types of fills. Another series of measurement was finding out an influence of a combination of two type of fills and two type fills, where one of them was covered by a hydrophobic surface, on the size of droplets in rain zone.

\subsection{Data evaluation}

The capture images from the camera were evaluated in Matlab. A first step was increased contrast to staying black background and white blobs. This process is simply because a signal from the measurement is great due to use the Rhodamine $\mathrm{G}$ and the green laser light. In the Fig. (6) is showed this process.

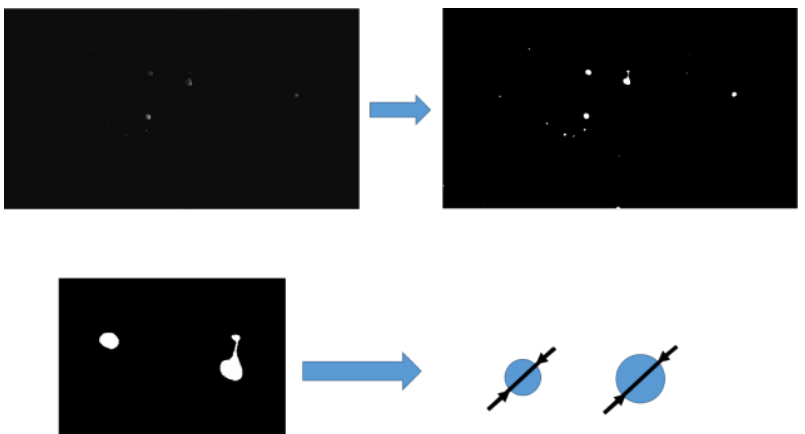

Fig.6. Process of droplets diameters evaluating

A second step is to calculate from the white blobs equivalent spherical droplet diameters $d_{i}$. Third step was a calculation of Sauter mean diameter (SMD) of all (N) calculated droplets from the equation:

$$
S M D=\frac{\sum_{i=1}^{N} d_{i}^{3}}{\sum_{i=1}^{N} d_{i}^{2}}
$$

The Sauter mean diameter allows a comparison of measured fills. When the Sauter mean diameter smaller, the efficiency of heat transfer is better in rain zone.

\section{Results}

Histograms of normalized droplets size were obtained from the evaluated data. In Fig. 7-11 are shown this normalized histograms. It is obvious that a change of droplets diameter is not very significant for different distances.

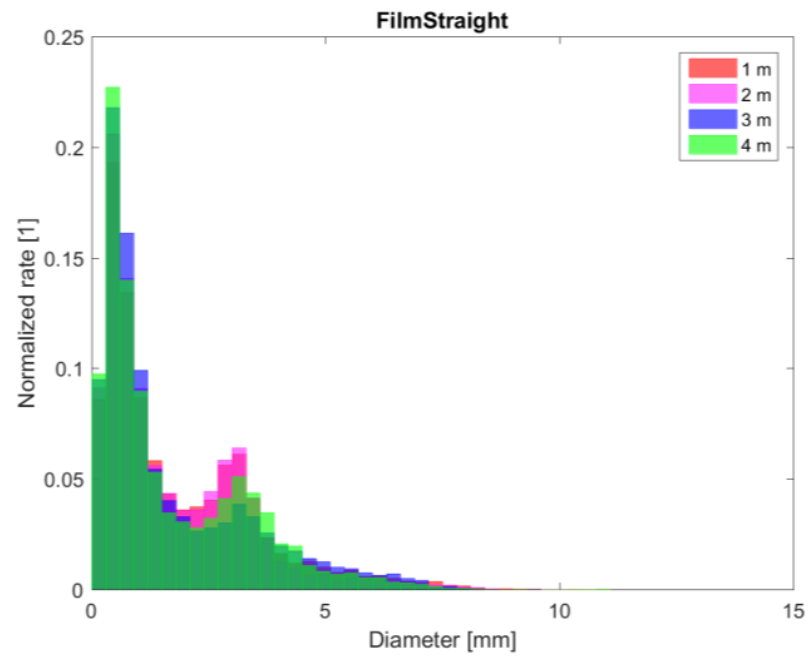

Fig. 7. Histogram of droplets diameters under film fill straight. 


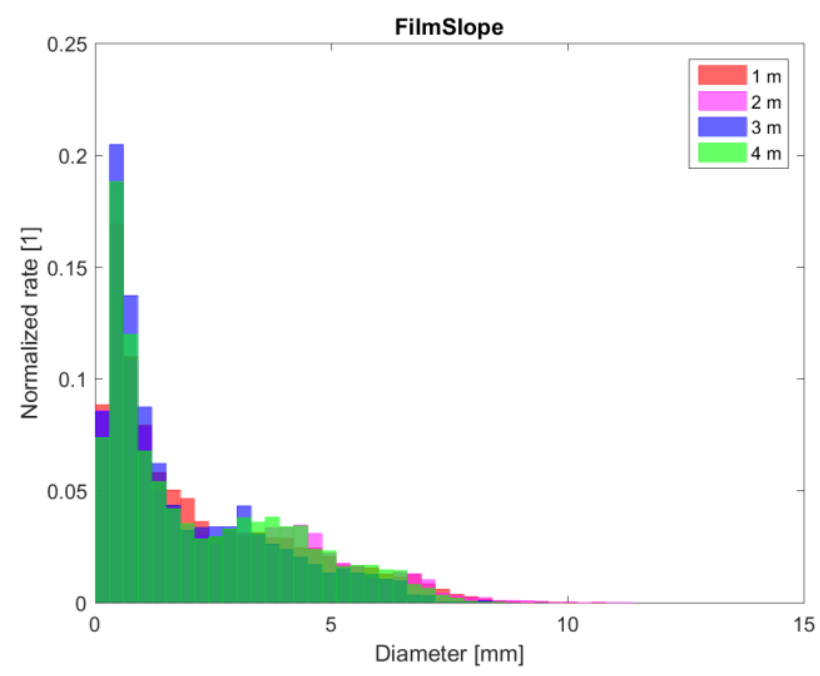

Fig. 8. Histogram of droplets diameters under film fill slope.

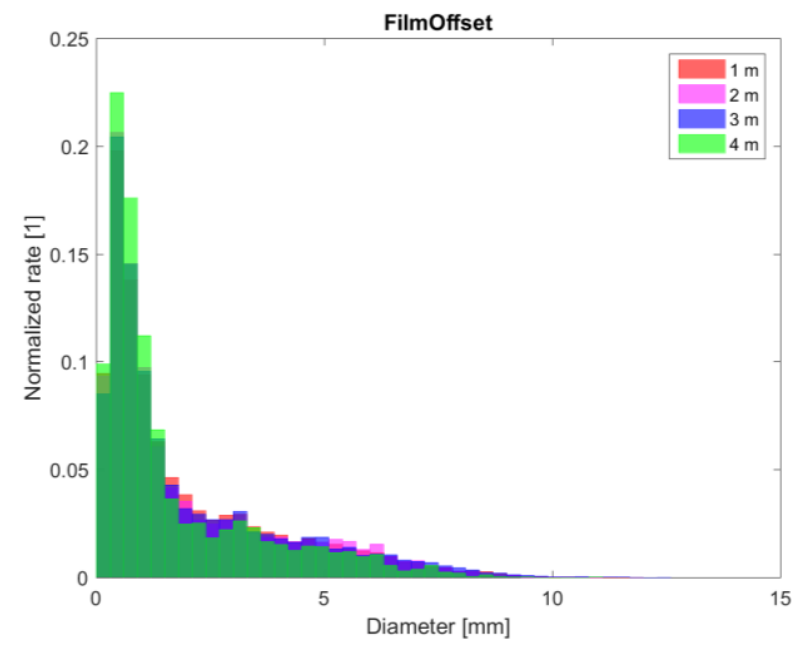

Fig. 9. Histogram of droplets diameters under film fill offset.

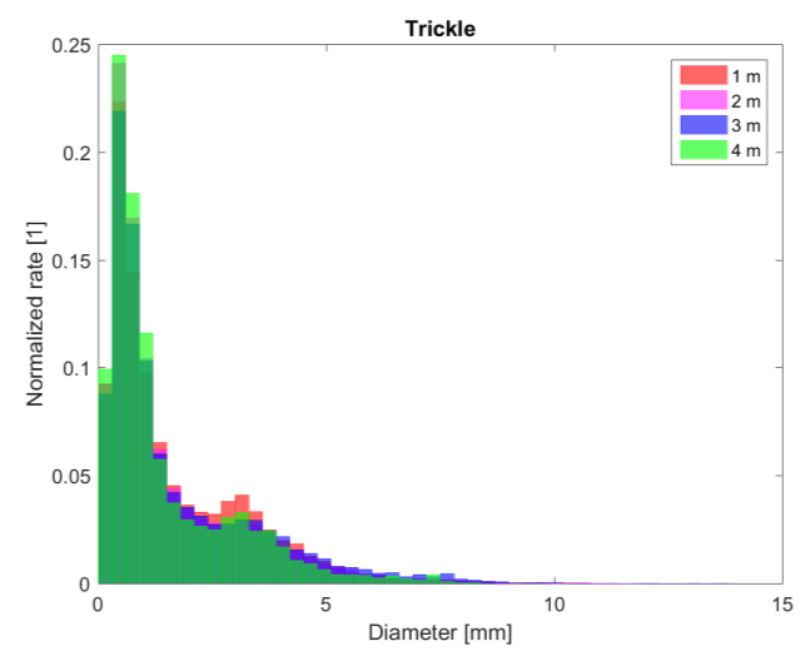

Fig. 10. Histogram of droplets diameters under trickle fill.

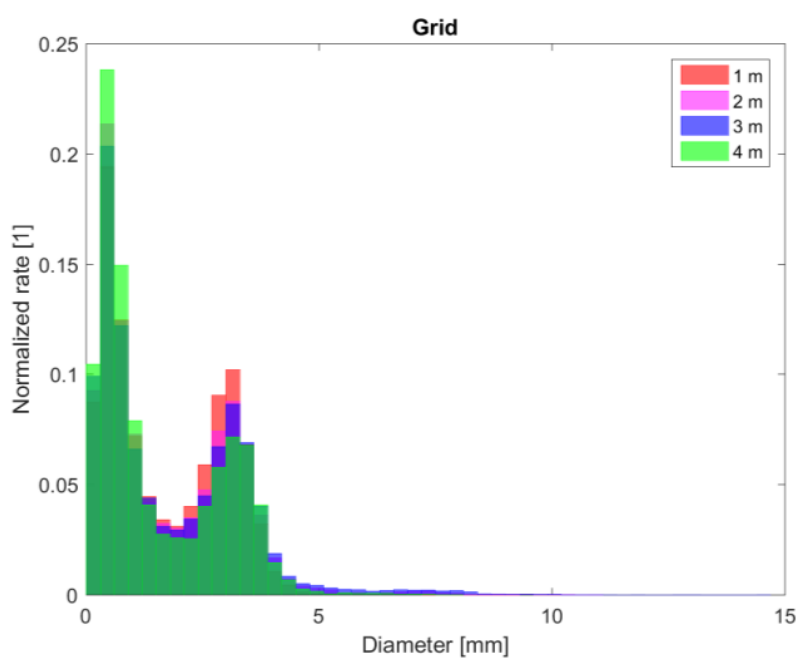

Fig. 11. Histogram of droplets diameters under grid fill.

It was calculated Sauter mean diameter for each fill. A comparison of Sauter mean diameter of this measured fills is in Tab. 1. The values of Sauter mean diameter show that the grid fill is the best fill for making a small droplets.

Table 1. Sauter mean diameter of each fill.

\begin{tabular}{|c|c|}
\hline Type of fill & Sauter mean diameter [mm] \\
\hline Straight film fill & 4.23 \\
\hline Slope film fill & 4.87 \\
\hline Offset film fill & 5.26 \\
\hline Trickle fill & 4.37 \\
\hline Grid fill & 3.52 \\
\hline
\end{tabular}

Based on previous experiments, theoretical analysis and analysis of a formation and a breakup of droplets $[7,8,9,10,11]$, a combination of two fills was chosen to improve cooling performance in the rain zone while maintaining a high heat transfer efficiency directly on the fill. This option uses the splash mechanism (breaking a droplet on satellite droplets). The film fill was selected as the main fill, and a grid fill placed under film fill was selected as a complement fill to the splash mechanism. Two variants of this combination were measured. The first variant was the combination of these fills without any modification, the second combination was a grid fill with a hydrophobic layer. Hydrophobic layer prevents the formation of water film on the surface and allows smaller droplets to form. For experimental work, the hydrophobic layer was made of wax (Fig.12). 


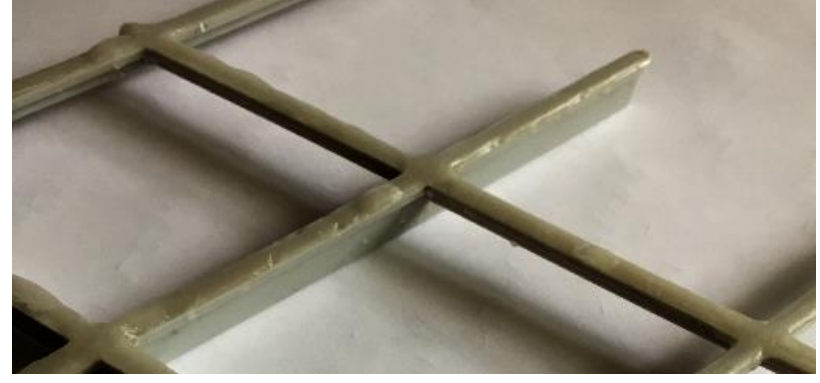

Fig. 12. Grid fill with hydrophobic surface.

Figures 13 and 14 show histograms comparing the rate of droplets between a combination of film fill and grid fill with wax with film fill itself (Fig.13) and film fill and grid fill with wax and fill and grid fill without surface treatment (Fig.14).

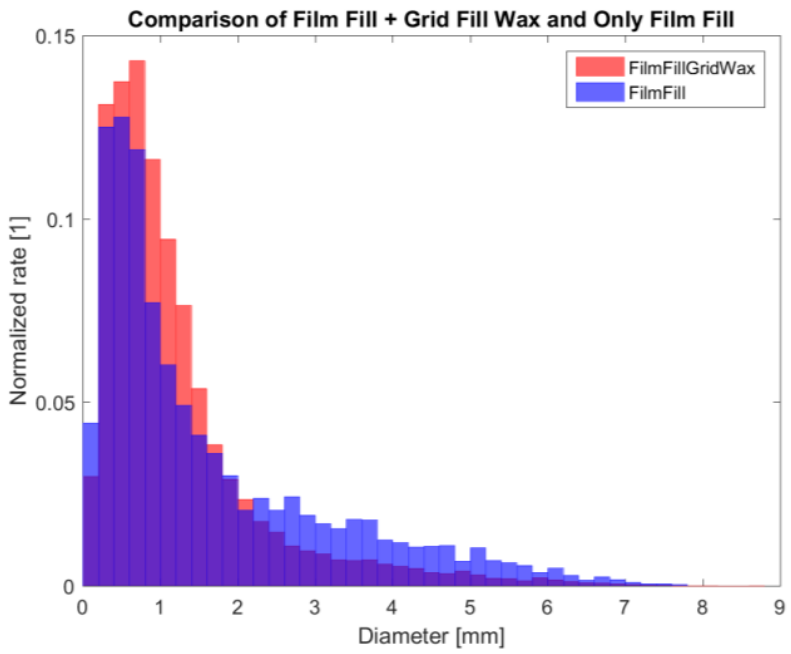

Fig. 13. Histogram of comparison combinations of film fill and grid fill with wax with film fill itself.

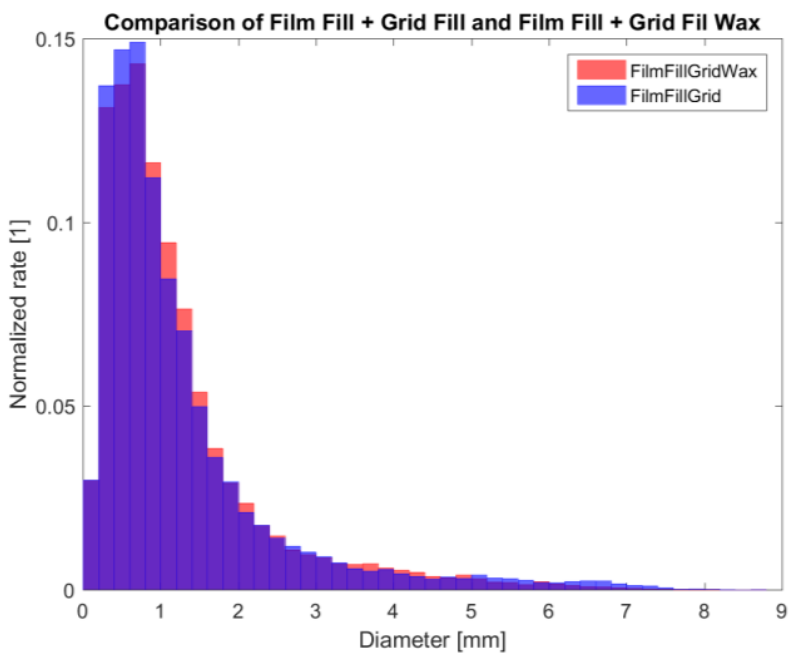

Fig. 14. Histogram of comparison combinations of film fill and grid fill with wax and fill and grid fill without surface treatment.
As is clear from the histograms, the film fill and grid fill combination with wax greatly reduces droplets in the rain zone to the film fill itself. The difference between the combination of film fill and grid fill without surface layer and the film fill and grid fill with wax is not very significant (Sauter mean diameter difference is about 0.5 $\mathrm{mm}$ ), but it can be noticed that the grid fill with wax does not make nearly any droplets larger than $6.5 \mathrm{~mm}$, but at the grid fill the maximum diameter is $7.5 \mathrm{~mm}$. This is due to the limitation of drip mechanism of grid fill with the hydrophobic layer. The Sauter mean diameters comparison is in Tab. 2, where the Sauter mean diameter for the droplet generator itself is mentioned for a comparison.

Table 2. Sauter mean diameter of combinations.

\begin{tabular}{|c|c|}
\hline Type of fill & $\begin{array}{c}\text { Sauter mean diameter } \\
\text { [mm] }\end{array}$ \\
\hline Droplet generator (DG) & 2.79 \\
\hline $\mathrm{DG}+$ film fill & 4.87 \\
\hline $\mathrm{DG}+$ film fill + grid fill & 3.90 \\
\hline $\begin{array}{c}\mathrm{DG}+\text { film fill }+ \text { grid fill } \\
\text { with wax }\end{array}$ & 3.37 \\
\hline
\end{tabular}

\section{Conclusion}

From the droplet size measurements at four distances below the fill, it appeared that the droplet size range under the fill doesn't change much with a distance, but only a small increase in the number of smaller droplets with a greater distance from the fill can be observed. If we compare the Sauter mean diameters, it is clear, as already mentioned, that the grid fill makes the smallest droplets. The reason is the predominant splash effect over dripping. The film fill straight and the trickle fill have the Sauter mean diameter of approximately the same value. This may be due to the fact that some droplets pass the fill straight through the channel without touching the walls of the fill. The trickle fill has a larger Sauter mean diameter against to the grid fill, because on the surface of the trickle fill the water film is formed to a greater extent and therefore it occurs more the drip mechanism. The largest drops are made by the film fill offset. The reason may be the dominating drip mechanism.

For a conservation of a high efficiency of heat transfer on the fill but also to increase efficiency in the rain zone, based on these previous measurements and theoretical analysis, a solution was chosen where a grid fill for small droplets was added under highly effective film fills. It was also found out to what extent the improvements will occur if this fill is covered with a hydrophobic layer. It is clear from the presented results that the grid fill placed under the film fill will greatly contribute to the reduction of droplets diameter in the rain zone. The difference between the standard and grid fill with the hydrophobic surface is not so obvious, 
however, it can be seen that the hydrophobic surface prevents more water film formation and reduces the drip mechanism and supports the spalsh mechanism. The results show that in cooling towers where film fill can be used, the efficiency of cooling can be improved by adding an additional build in order to minimize pressure loss.

\section{Acknowledgments}

The author acknowledges the support received from Advanced technologies for heat and electricity production TE01020036 and Centre of 3D volumetric velocimetry - COLA supported by the European Union (CZ.2.16/3.1.00/21569) Operating Program Prague competitiveness.

\section{References}

1. D.G. Kröger, Air-Cooled Heat Exchangers and Cooling Tower, (2004)

2. H.C. Reuter, D.G. Kröger ASME IHTC, 4, 619 628, (2010)

3. T. Hyhlík, Engineering Mechanics, 21, 240-243, (2014)

4. T. Hyhlík, ICCT 2014 International Conference, 219-226, (2014)

5. R. N. Zare, Annual Review of Analytical Chemistry, 5, 1-14, (2012)

6. P. Vitkovic, EPJ Web of Conferences, 114, 02137, (2016)

7. A. L. Yarin, Annu. Rev. Fluid Mech. 38, 15992,(2006)

8. A. A. Dreyer, P.J. Erens, J. Heat Mass Transfer, 39, 109-123, (1996)

9. C. Josserand, S. T. Thoroddsen, Annu. Rev. Fluid Mech., 48, 365-91, (2015)

10. P. Vitkovic, EPJ Web of Conferences, 143, 02142, (2017)

11. M. Rein, Fluid Dyn. Research, 12, 61-93, (1993) 